- Bolinder J. Novel glucose-sensing technology and hypoglycaemia in type 1 diabetes: a multicentre, non-masked, randomised controlled trial. Lancet 2016; 6736(16):1-10.)

- DanneT. International Consensus on use of continuous glucose monitoring. Diabetes Care 2017; 40:1631-1640.

- American Diabetes Association. Standards of medical care. Diabetes Technology. Diabetes Care 2019; 42(Suppl.1):S1-S.

\title{
Nuevos infusores
}

Dr. Alejandro Daín

Acompañando la gran revolución tecnológica de los últimos años, los infusores de insulina no han sido la excepción'. Desde sus orígenes, en poco más de 50 años, estos equipos, que cambiaron la vida de muchas personas con diabetes y su familia, experimentaron diferentes avances: mejores técnicas de cambio de reservorio y manejo de los set de infusión, calculadoras de bolo que incorporan funciones avanzadas o sistemas mecánicos con rapidez en la detección de oclusión, entre otros². En los últimos años, el desarrollo de métodos de monitoreo continuo de glucosa (MCG) asociados al infusor, con sistema de corte predictivo frente a la tendencia en hipoglucemias, ha sido un importante avance. En la actualidad, en Argentina disponemos de dos compañías que aportan experiencia en la promoción de estos productos: Laboratorio Roche ${ }^{\circledR}$ y Medtronic ${ }^{\circledR}$. Próximamente contaremos con novedades, productos que se agregarán a los sistemas Accu-chek Combo (Roche ${ }^{\circledR}$ ) y Minimed 640 (sistema integrado con MCG con corte predictivo de Medtronic $\left.{ }^{\circledR}\right)$ como los siguientes infusores:

- Laboratorio Roche ${ }^{\circledR}$ Accu-chek solo: esta infusora será la primera de su clase en el país. Una infusora sin tubuladura o catéteres (también llamados patch pumps) que se usa como un parche directamente en el cuerpo y se controla desde un glucometer touch screen de mano inalámbrica, lo que permite un suministro discreto de insulina. Su tecnología innovadora permitirá, con énfasis en la población pediátrica, una infusión de insulina sin las complicaciones asociadas a los set de infusión y cánulas, con mejores alarmas de oclusión y una programación segura de insulina ${ }^{3}$.

- Laboratorio Medtronic ${ }^{\circledR}$ Minimed 670G: esta infusora presenta una importante innovación que se suma al modelo anterior de $640 \mathrm{G}$ con todas sus ventajas en el combinado $\mathrm{SICl}+\mathrm{MCG}$, ser la primera infusora autoprogramable o sistema híbrido. Como características generales la infusora tendrá su basal autoprogramable según datos del MCG Guardian 3, que contará con alta exactitud con un MARD de $8,7 \%$. En definitiva, la persona con diabetes y su equipo médico no deberán programar basales; esto lo hará sólo la infusora simultáneamente al sensado continuo de glucosa intersticial. Estos sistemas nos acercan al concepto de páncreas artificial ${ }^{4}$.

De esta manera nuevas herramientas lograrán mejor calidad de vida, y mayor precisión y exactitud en la infusión de insulina que representarán, en las personas con diabetes mellitus tipo 1 y su entorno, un futuro sin complicaciones.

\section{BIBLIOGRAFÍA}

1. Pickup JC. Insulin pumps. Int J Clin Pract 2012; 66:15-9.

2. Shah VN, Shoskes A, Tawfik B, Garg SK. Closed-loop system in the management of diabetes: past, present, and future. Diabetes TechnolTher 2014; 16:4

3. Baghban-Taraghdari Z, Imani R, Mohabatpour F. A review on bioengineering approaches to insulin delivery: a pharmaceutical and engineering perspective. Macromol Biosci 2019.

4. Kovatchev B. Automated closed-loop control of diabetes: the artificial pancreas. Bioelectron Med 2018; 4:14.

\section{Evidencia disponible}

Dra. Graciela Rubín

La tecnología de la diabetes es el término que se usa para describir el hardware, los dispositivos y el software que usan las personas con diabetes para controlar sus niveles de glucosa en sangre, evitar las complicaciones de la diabetes, reducir la carga de vivir con la enfermedad y mejorar la calidad de vida. Dada su gran trascendencia y desarrollo, por primera vez este año la American Diabetes Association (ADA) incluye en su suplemento anual de "Estándares de atención médica en la diabetes" un capítulo destinado a la misma. 